not only exists, but is transmissible

from place to place by means of clothes, $\&_{c}$. The experiment would also, he observes, determine pretty well the efficacy of quarantine and sanatory codes. n the other hand, if the experimenwis remain free from the disease, notfocidanding all these exposures to the

that nidi of contagion, he imagines sion way safely come to the conclusion that cholera morbus is not propagated by infected materials.

However well adapted, the foregoing experiment might be for ascertaining the important point in question, it is perfectly evident that the French Government will not adopt the proposal. If all France were savans and physicians, the plan might be put in execulearn. But the prejudices of the unand community must be respected; of we imagine that no small portion of this, the largest class in all countries, would protest against the measure, as a wanton, nay, an impious inthoduction of a pestilence which it was all duty of Government to exclude, by all possible means, from the territuries over which they bore sway.

P.S. The Government has politely re-
fused.

\section{Silit isher:}

\section{axifo certe XXXI.}

Ovarian Tumour cured by Puncture and IRritating Injections.

A CASE of this kind is detailed in the Transactions of the Medical Society of Lyon, by M. Rigollot. The patient was 23 years of age, who experienced, after an accouchement, an attack of uterine inflammation, that was not very skilfully treated. The disease became chronic, attended with dull settled pain in the ovarian region, and general ailing. The abdomen became swollen, and slow consumptive fever supervened. deriting medicines administered (under the supposition that the disease was tympanitis) aggravated materially all its symptoms. When M. Rigollot was called to examine the patient, her emaciation was extreme, her fever continued, the abdomen prominent in its ante- rior and left lateral part, and obscure fluctuation was perceptible. The tumour was punctured, and ten or twelve pounds of purulent, fotid, and greenish fluid, escaped through the canula.

Q. Twenty days after, a second puncture was made, which gave issue to some matter, and the cavity was then injected with a decoction of plantain and red rose leares, with a little wine. Acute pain was endured while the injection remained in the cyst. After its entire discharge, the surgeon kneaded the cyst with his fingers, in order to determine its inflammation. Intense pain, vomiting, and swelling of the abdomen quickly ensued, and were energetically treated with antiphlogistic measures. In a month the cure was complete. The patient, nevertheless, retained in the abdomen a small, oblong indolent tumour, doubtless formed by the adherent parietes of the cyst.

We would caution junior and "bold surgeons" against too confident expectations from tapping and injecting ovarian cysts. We have witnessed some of these operations ; but the results were not of an encouraging nature. When we reflect on the well known fact, that these cysts are almost aliways divided into compartments, having little or no communication with each other, it will be evident that we cannot evacuate them by puncture; and that to excite inflammation in one or two of these compartments will only tend to increase effusion into the others. Besides, the inflammation itself of the cyst is no trifling danger. In most of the operations which we have witnessed, the patients died of this inflammationeven from common puncture. Within these few days (5th August) we witnessed a fatal case of inflammation of the cyst of an ovarian tumour without any operation; and as the case is curious we shall here state a few of the particulars. The patient was a lady about 35 or 38 years of age, who had a tumour in the centre of the abdomen, which was perfectly circumscribed, excepting inferiorly. The early history of the case could not be obtained, and therefore it was not ascertained whether or not the tumour originated in 
one side of the abdomen. Two or three times this tumour appeared to be the seat of acute inflammation, and required very active depletion. A similar attack nccurred early in August last, and Mr. Skair, of Castle-street, employed the same means as formerly used, viz. copious depletion, but without the same success. Instead of a speedy cessation of pain, as formerly the result of venesection, the patient fell into a state of exhaustion, with laborious breathing, cold extremities, and great restlessness. From this state she could not be roused by cordials, and she died in less than 24 hours. Mr. Thomas, Mr. Skair, and Mr. Nicholson, examined the body, and we were present at the examination. The tumour resembled a uterus in the sixth or seventh month of pregnancy. It was almost black, so completely injected were the vessels of the cyst. In short, it looked as if it were in a state of incipient gangrene. Yet, strange to say, there was not the slightest adhesion between it and the surrounding intestines, nor any effusion of fluid in the abdomen. No other part was inflamed except the sac of the ovarian tumour, which rose by a very narrow peduncle from one of the fallopian tubes. The other ovary was a solid scirrhus, the size of a smalt apple, and the uterus itself was scirrhous. The cyst contained several compartments, most of which were filled with a fluid resembling blood and water. The others contained solid and semi-solid contents of various kinds.

This case shews that an inflamed cyst may occasion death, without the inflammation spreading to any other organ or part. Therefore the exciting of inflam. mation in an ovarian cyst is a matter of no trifling danger. The circumstance of the ovarian tumour having gained such a size without any adhesions, and also the fact of its being connected, up to the period of death, by only a small peduncle with the uterus, are favourable to the proposals of those who have attempted and recommended extirpation of diseased ovaries. No case could be more favourable for such an operation than this, as far as the execution of the operation is concerned:- and yet it would have been unsuccessful in the end on account of the scirrhous disease of the other ovarium and of the uterus - diseases which would, no doubt, have been called into activity by such a formidable operation as gastrotomy.

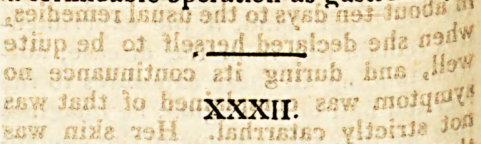

SUPPOSED CASE OF INFlammation of The Pancreas.* * olos srdas

If little is known respecting the morbid affections and conditions of the pancreas, it is partly because the gland, like the other salivary glands, is little prone to disease ; and partly because its sititation and other circumstances have conspired to render examinations of its state after death comparatively unfrequent. Intlammation of the pancreas has not been described by Baillie, Meckel or Andral. Portal pronounces it of com mon occurrence, but probably the is sertion is not founded on satisfactory grounds. The following case is looked upon by Mr. Lawrence as an instance of the affection in question. Mr. $\mathrm{L}$. was not requested to see the patient until thirty-six hours before her death; she was attended during the progress of the disease by a very intelligent $\mathrm{ge}^{\mathrm{e}}$ neral practitioner and two physicians. The history of the case is given in the words of the former, nor do we see that it admits of abridgement.

Case. "At the time Mrs. married, she appeared to be in good health. When she was between five and six months advanced in pregnancy, she lost her usual healthy appearance, and gradually became very pallid. This change I observed on occasionally meeting ber in her walks from her own to her mother's house, and on enquiring generally after her liealth, her answer invariably was, 'I am quite well.'

About a month previous to her cunfinement, I, for the first time, was desired to see her professionally. She was then suffering from a severe attack

* Med, Chir. Trans. Vol. XVI. Part I. 